\title{
Research on the Risk Assessment of Food Cold Chain Logistics Based on Entropy Weight and Fuzzy Comprehensive Evaluation Model
}

\author{
Yun-Mei LI \\ Center for Industrial Policy and \\ Management Research, Wuhan \\ University of Science and Technology, \\ 123-116-2,Gangdu Garden, Qingshan \\ District, Wuhan, China \\ Email: lymwust@163.com,
}

\author{
Yong LUO* \\ Wuhan University of Science and \\ Technology, No.947, Heping Avenue, \\ Qingshan District, Wuhan, China \\ Email: luorenyong@foxmail.com
}

\author{
Ting ZOU \\ Wuhan University of Science and \\ Technology, No.947, Heping Avenue, \\ Qingshan District, Wuhan, China
}

\begin{abstract}
In order to evaluate the risks of food cold chain logistics, this paper begins from the component links of the food cold chain logistics system, and conducts researches based on the relevant theories of food cold chain logistics. First of all, the indexes of the food cold chain logistics risk assessment are selected and index weights are determined in accordance with entropy weight method. Then, we contract a risk assessment index system of food cold chain logistics based on entropy weight fuzzy comprehensive evaluation. Finally, representative food cold chain logistics enterprises are chosen for empirical analysis. The analysis results verify the effectiveness of the proposed model, providing a new research perspective towards the risk assessment of food cold chain logistics.
\end{abstract}

Keywords-Food cold chain logistics, Entropy weight method, Fuzzy comprehensive evaluation method, Risk assessment.

\section{INTRODUCTION}

With the continuous development of social economy and the improvement of living standards, the demand for cold chain food is increasing day by day. At the same time, there are some factors driving the rapid development of food cold chain logistics in China, such as the continuous refinement of social division of labor, the continuous progress of cold storage technology and the gradual popularization of low-temperature products. Although the development trend of the food cold chain logistics in China is good, we should notice the facts that food cold chain logistics is still subject to high cost in the process of development, it is difficult to coordinate every link and that the business environment is very complex. There are many risks in the food cold chain logistics industry. Meanwhile, the contradictions between the rapid development of food cold chain logistics industry and lagging relevant theoretical research is also increasingly highlighted. Thus, under this background, doing research on the risk assessment of food cold chain logistics has a certain theoretical and practical application value.

\section{LITERATURE REVIEW}

By reading the relevant literature, we found that the current literature focus on the risk research of normal temperature logistics, there is less risk research specifically for food cold chain logistics. Fang Xiaobo divided cold chain logistics system into five subsystems, including manufacturers, logistics center, retailers, control system and the external environment, and he regarded them as the factors of risk analysis for the influencing factors of the failure of cold chain logistics [1]. Quantitative evaluation about the business risk assessment to undertake the road freight industry of cold chain logistics was made by Yan P, Qin H, using fuzzy comprehensive evaluation method, in order to take effective risk management techniques to solve the derivative problems[2].Song Shaowen identified the best risk management solutions to realize the optimization on risk management and control. Through analyzing the cycle logistics chain risk, regarding controlling risk as the target layer, maintaining the risk factors that affect cost as the criterion layer[3]. Li Li, Liu Jian et al. constructed the logistics enterprise management risk early warning model based on confirming the logistics enterprise management risk early warning index system[4]. Hou Bin, Wang Yanrong et.al determined the weight of each index of risk factor in the joint distribution in urban logistic by using triangular fuzzy number method, they also used fuzzy comprehensive evaluation method to establish a comprehensive evaluation of risk factors of the matrix. Conclusions about the risk profile of the joint distribution of the city are reached [5].OuYang Liping puts forward that logistics management personnel can control and regulate the corresponding risk factors based upon received information, in the adjustment process they can use negative feedback control mechanism to adjust the risk factors and then take corresponding risk control measures, to make the degree of risk loss down to the lowest [6]. Liu Yongsheng selected a series of logistics risk assessment indicators, and establish enterprise logistics risk early warning model by using principal component analysis method, so a new method was provided for manufacturing listing Corporation to do a good job in the logistics risk early warning[7].

In summary, as described above, enterprise logistics risk assessment and early warning research at home and abroad has made some achievements, but research about the food cold chain logistics risk is not much, especially contraposing the food cold chain logistics enterprise risk assessment is rare. In the case of the development risk of 
cold chain logistics highlights, the food cold chain logistics enterprises need to recognize and identify potential risks and grasp the correct risk assessment methods and means. Based on this, this paper integrate entropy method and fuzzy comprehensive evaluation method, using the scientific system of standard, objective data to evaluate the risk of food cold chain logistics, improve the food cold chain logistics industry risk prevention awareness, and promote the healthy and steady development of food cold chain logistics industry.

\section{ESTABLISHMENT OF VALUATION INDEX AND RISK ANALYSIS OF FOOD COLD CHAIN LOGISTICS}

\section{A. Risk Factors Analysis of Food Cold Chain Logistics}

As the cold chain logistics starts late, the development is not perfect, and its development is more and more restricted by a series of factors. First, cold chain infrastructure is lacked. A serious lack of cold chain facilities and cold chain equipment makes cold chain food that should have been under low temperature distribution can't be guaranteed low temperature distribution security. There are many cold chain food in the open air, instead of loading operation in cold storage and holding areas, and mostly shipped by ordinary truck. Second, the scale is not enough. Most of the cold chain logistics enterprises are unable to extricate themselves from the whirlpool of price competition, and the profit is too weak to expand production, which directly inhibits the scale development of food cold chain logistics industry. Third, technical standards are not perfect. Food cold chain logistics, as a special kind of supply chain, its purpose is to protect perishable food quality and safety, so it requires more than the general logistics systems, more complex management, more construction investment. And the current domestic cold chain system is only a preliminary market, there are a large gap compared with the international advanced level. At the same time, the lack of national or industry specific standards in the implementation of the cold chain is a problem, and relevant laws and regulations have yet to be improved. Fourth, lack of professional reserve. The rapid development of the food industry drives the rapid expansion of the food cold chain logistics industry, but at the same time there is serious disjunction in the personnel training, so training professional personnel of cold chain logistics has become one of the problems that needs to be solved urgently in the development of cold chain logistics. Fifth, information leakage. Because the information sharing requirements in food cold chain logistics is higher than other logistics, the enterprise information is easy to disclosure if supervision is not enough, which will bring a fatal blow to the development of enterprises.

\section{B. Food Cold Chain Logistics Risk Evaluation Index System}

By reference to the domestic and foreign relevant risk assessment literature, basing upon the actual situation of food cold chain logistics industry, and the index selection principle, including scientific and systematic, logical and operation, we construct the initial risk evaluation index system of food cold chain logistics. Then we consult the opinions of the experts of the index system to adjust and improve. Eventually, the food cold chain logistics risk evaluation index system is established, including the external environment risk, market risk, facility risk, human resource risk and technology risk, the index system is consist of 5 first class indexes and 13 secondary indicators, as shown in Fig. 1.

\section{FUZZY COMPREHENSIVE EVALUATION MODEL BASED ON ENTROPY WEIGHT}

\section{A. Using Entropy Weight Method to Determine Index Weight}

Entropy is one of the core concepts in statistical physics and information theory, but the concept of entropy has been widely used in different subject areas now [8]. The use of entropy method to determine index weight in this paper has its objectivity and rationality. In the previous process of determining the index weight, AHP and some subjective methods are commonly used to determine the weight, but because of subjective factors, evaluation results deviate. This paper uses entropy evaluation method to analyze and deal with the results of subjective weight objectively, so as to eliminate the jamming of the weight in each index, making the evaluation results more objective. According to the entropy weight method, the risk assessment index weight vector set can be obtained based on the entropy weight, namely, $\omega=\left(\omega_{1}, \omega_{2} \ldots \omega_{\mathrm{n}}\right)$.

\section{B. Fuzzy Comprehensive Evaluation Model of Entropy Weight}

Mainly taking into account the food cold chain logistics risk evaluation of multi index is a quantitative, and cannot use general method for direct comparison, in this paper, we use fuzzy comprehensive evaluation method which exactly suited to deal with fuzzy information in a fuzzy environment to analyze [9]. Fuzzy comprehensive evaluation method is the application of fuzzy synthetic relationship principle, by constructing grade fuzzy subset quantization was rated the fuzzy index of things, it is a quantitative evaluation method make a comprehensive evaluation about membership degree from multiple angles [10]. The specific steps are as follows:

Determine the set of factors to be evaluated

Assume that the objective being evaluated contains $n$ factors, i.e. ,the index set is $U=\left(\mathrm{u}_{1}, \mathrm{u}_{2} \ldots \mathrm{u}_{\mathrm{n}}\right)$.

Determine the set of evaluation object comment

The appraisal setV $=\left(\begin{array}{llll}\mathrm{v}_{1} & \mathrm{v}_{2} & \ldots & \mathrm{v}_{\mathrm{n}}\end{array}\right)$ is the possible evaluation grading result. Each level can correspond to a fuzzy subset.

Constructing fuzzy relation matrix

After determining the level of fuzzy subset, it is to be evaluated one by one on each factor to quantify, and then get the fuzzy relationship matrix:

$$
\mathrm{R}=\left[\begin{array}{cccc}
\mathrm{r}_{11} & \mathrm{r}_{12} & \ldots & \mathrm{r}_{1 \mathrm{~m}} \\
\mathrm{r}_{21} & \mathrm{r}_{22} & \ddots & \mathrm{r}_{2 \mathrm{~m}} \\
& \vdots & & \ddots \\
\mathrm{r}_{\mathrm{n} 1} & \mathrm{r}_{\mathrm{n} 2} & \cdots & \mathrm{r}_{\mathrm{nm}}
\end{array}\right](1)
$$

The i-th row $j$-th column element $r_{i j}$ in matrix 
Rrepresents a factor $\mathrm{u}_{\mathrm{i}}$ been assessed from the point of view of things fuzzy subset membership of $\mathrm{v}_{\mathrm{j}}$ level. An assessment of things in terms of a performance factor, is portrayed by fuzzy vectors, while in other evaluation methods is more an indicator of the actual value to portray, so from this perspective, fuzzy comprehensive evaluation requires more information.

Determine the weight of evaluation factors

In the fuzzy comprehensive evaluation, to determine the weight vector of evaluation factors: $\omega=\left(\omega_{1}, \omega_{2} \ldots \omega_{n}\right)$. The author uses the entropy weight method to determine the relative importance of evaluation indicators, so as to determine the weight, and normalized before the synthesis.

Synthetic fuzzy synthetic evaluation result vector

Synthesizing fuzzy weight vector and fuzzy relationship matrix $\mathrm{R}$, we obtain the results of the evaluation object vector $\mathrm{U}$. That is

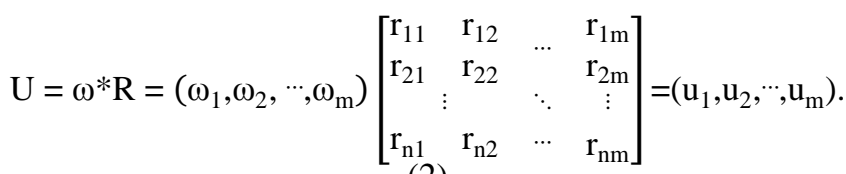

Among them, $\mathrm{u}_{\mathrm{m}}$ said that the evaluation of things from the overall perspective on the $v_{i}$ level of fuzzy subset of the degree of membership.

\section{V.EMPIRICAL ANALYSIS}

This paper selects food cold chain logistics listed $Y$ enterprise to do risk analysis, to provide scientific basis of the enterprise risk assessment activities, according to the evaluation index system in figure 1, we use the entropy weight fuzzy comprehensive evaluation model to analyze the degree of risk.

\section{A. Determine the Weight of Each Layer Index Set}

Fig.1 shows that the food cold chain logistics risk evaluation index system,according to the enterprise leadership and research of cold chain logistics experts invited from a total of five on each index of relative importance of scoring, scoring range is on a scale of 1-10. According to the calculation rules of the entropy weight method, the relative weight vector of the first layer is obtained. Weight vector of $U_{i}$ to $U$ layer $\omega=\left(\omega_{1}, \omega_{2}, \omega_{3}\right.$, $\left.\omega_{4}, \omega_{5}\right)=(0.131,0.276,0.317,0.121,0.155)$; by the same method, the weight vector of $U_{i j}$ to the $U_{j}$ layer is obtained: $\omega_{1}=(0.36,0.35,0.29)$,

$\omega_{3}=(0.407,0.262,0.391)$, $\omega_{2}=(0.171,0.372,0.457)$, $\omega_{5}=(0.633,0.367)$.

\section{B. Fuzzy Comprehensive Evaluation of Food Cold Chain Logistics}

Determine the set of factors to be evaluated

$\mathrm{Y}$ food cold chain logistics enterprise evaluation index of the domain can be specifically expressed as: $U=\left\{U_{1}, U_{2}, U_{3}\right.$, $\left.\mathrm{U}_{4}, \mathrm{U}_{5}\right\} ; \mathrm{U}_{1}=\left\{\mathrm{U}_{11}, \mathrm{U}_{12}, \mathrm{U}_{13}\right\} ; \mathrm{U}_{2}=\left\{\mathrm{U}_{21}, \mathrm{U}_{22}, \mathrm{U}_{23}\right\} ; \mathrm{U}_{3}=$ $\left\{\mathrm{U}_{31}, \mathrm{U}_{32}, \mathrm{U}_{33}\right\} ; \mathrm{U}_{4}=\left\{\mathrm{U}_{41}, \mathrm{U}_{42}\right\} ; \mathrm{U}_{5}=\left\{\mathrm{U}_{51}, \mathrm{U}_{52}\right\}$.

Determine comment hierarchy

According to the degree of influence of our target, we set up a grade evaluation set as follows: $V=\{$ "safe", "low risk", "medium risk", "high risk", "extreme risk” $\}=(0.2,0.4,0.6,0.8,1)$

Determine Fuzzy relation matrix

Delphi method is used to determine membership degree of the evaluation set, and then make the fuzzy judgment matrix, such as

$$
R_{1}=\left[\begin{array}{ccccc}
0.4 & 0.3 & 0.1 & 0.3 & 0.3 \\
0.1 & 0.1 & 0.2 & 0.3 & 0.3 \\
0 & 0.1 & 0.2 & 0.3 & 0.4
\end{array}\right]
$$

Results of comprehensive evaluation

The comprehensive fuzzy judgment result is calculated $\operatorname{asB}_{\mathrm{i}}=\omega_{\mathrm{i}} \times \mathrm{R}_{\mathrm{i}}$, that is

$$
\begin{aligned}
& \mathrm{U}_{1}=\omega_{1} \times \mathrm{R}_{1}=(0.1790 .1720 .1640 .300 .329)(3) \\
& \text { Similarly, we know that: } \mathrm{U}_{2}= \\
& \text { (0.1020.1760.1790.4680.185), } \quad \mathrm{U}_{3}= \\
& (0.040 .1350 .270 .180 .455) \\
& \mathrm{U}_{4}=\quad(0.0650 .3340 .310 .2230 .235), \quad \mathrm{U}_{5}= \\
& \text { (0.0370.1630.2270.100.209). }
\end{aligned}
$$

$Y$ food cold chain logistics enterprises for the evaluation set of the degree of membership:

$\mathrm{U}=\omega \times \mathrm{R}=(0.0780 .180 .2290 .2680 .299) .(4)$

Fuzzy comprehensive risk assessment results

From the above we can know:

$$
\mathrm{C}=\mathrm{U}^{*} \mathrm{~V}^{\mathrm{T}}=\left(\begin{array}{llll}
0.0780 .18 & 0.229 & 0.268 & 0.299
\end{array}\right) *\left(\begin{array}{llll}
0.2 & 0.4 & 0.6
\end{array}\right.
$$$$
0.81)^{\mathrm{T}}=0.738 \text {. }(5)
$$

Therefore, the system risk level of food cold chain logistics enterprise $\mathrm{Y}$ is $0.738<0.8$, according to the evaluation set, the risk status of the enterprise is between the medium risk and high risk. The first level indicators: market risk rating for "medium risk", technology risk rank for "medium risk", equipment risk as "high risk", the risk of human resources for "medium risk", environmental risk is "low risk". From the evaluation results we can see that the cold chain logistics risk of the enterprise overall in the middle level, in which the risk of facilities and equipment is higher. So it is necessary to increase the investment and maintenance of cold chain facilities and equipment. In addition, the company still need to take certain measures to control the market risk and technical risk, and strengthen the cold chain logistics talent reserves.

\section{CONCLUSION}

Food cold chain logistics enterprise in development may be affected by all kinds of potential risks. The process of risk research is to analyze risky factors that have influence on enterprise development, which makes it easier for policymakers to get the related risky information more timely and accurately, to quickly capture all possible factors that induce enterprise crisis and to take the corresponding preventive measures to reduce unnecessary losses. This thesis analyzes risky factors of developing food cold chain logistics industries, constructs the index system of risk assessment on food cold chain logistics, and uses entropy theory to calculate the corresponding weight coefficient of various risk factors and establish the entropy weight fuzzy comprehensive evaluation model of food cold chain logistics enterprise risk evaluation, so it achieves a better 
effect of evaluation. The relevant business of risk assessment, based on the value in evaluation of the results of the risk assessment, is helpful for the decision-makers to overall grasp the risk status of the food cold chain logistics industry, to timely understand the problems existing in the development of the enterprise, and to provide a reference for the improvement and evaluation of food cold chain logistics enterprise risk management. It is more objective to introduce the entropy weight method, but there are still problems that need attention to be improved. For example, the modelling process is complicated, of large number calculations, and inevitably exists some subjective factors.

\section{ACKNOWLEDGEMENT}

This research was financially supported by Center for Industrial Policy and Management Research of Wuhan University of Science and Technology.

\section{REFERENCES}

[1] Fang Xiaobo, Identification of Risks for Cold Chain Logistics Failure, J. Technoeconomics \& Management Research. 2015(8):26-30.

[2] Yan P, Qin H. The assessment research of operational risk in the road freight industry undertake cold chain logistics services[C].International Conference on Remote Sensing, Environment and Transportation Engineering. IEEE, 2011:1757-1760.
[3] Song Shaowen. Establishment of recycling logistics chain and risk management and control model, J. Commercial Times. 2016(7).

[4] Liu Jian, Lili, Guan Yuhang Yuan Dejiang. An Empirical Study on the Formulation of Logistics Enterprise Operational Risk Warning Model, J. Logistics technology. 2010(2):59-62.

[5]Bin Hou, Wang Yanrong, Dan Miyuan. Research on the Urban Joint Distribution Risk Evaluation Based on Fuzzy Comprehensive Judgement, J. Science and Technology Management Research, 2015,08:52-56+61.

[6] OuYang Liping. Application of Cybernetics in Logistics Outsourcing Risk Control, J. Journal of Social Sciences, 2016(1).

[7] Liu Yongsheng. On Logistics Risk Early Warning for the Listed Corporations of Manufacturing Industry in Beijing ——Based on Principal Components Analysis, J. China Business and Market, 2013, 27(4):60-66.

[8] Zhao Chengxuan, Panayiotis Papadopoulos, Li Jianyu, et al. An entropy-based evaluation of contact forces in continuum mechanics of elastic structures, J. Finite Elements in Analysis and Design, 2016, 114:78-84.

[9] Song Baoe, Zhu Wenying, Li Xiaoming. The Research on the Assessment of Farm Produce Cold Chain Quality and Safety Based on Fuzzy Comprehensive Evaluation, J.Journal of Food Science and Biotechnology,2013,10:1057-1062.

[10] Wang Zhongwei, Zhao Fangni.Study on the comprehensive evaluation of green cold chain logistics for agricultural products based on the fuzzy comprehensive evaluation method, J.Journal of Central China Normal University(Natural Sciences), 2015, 04:546-550. 


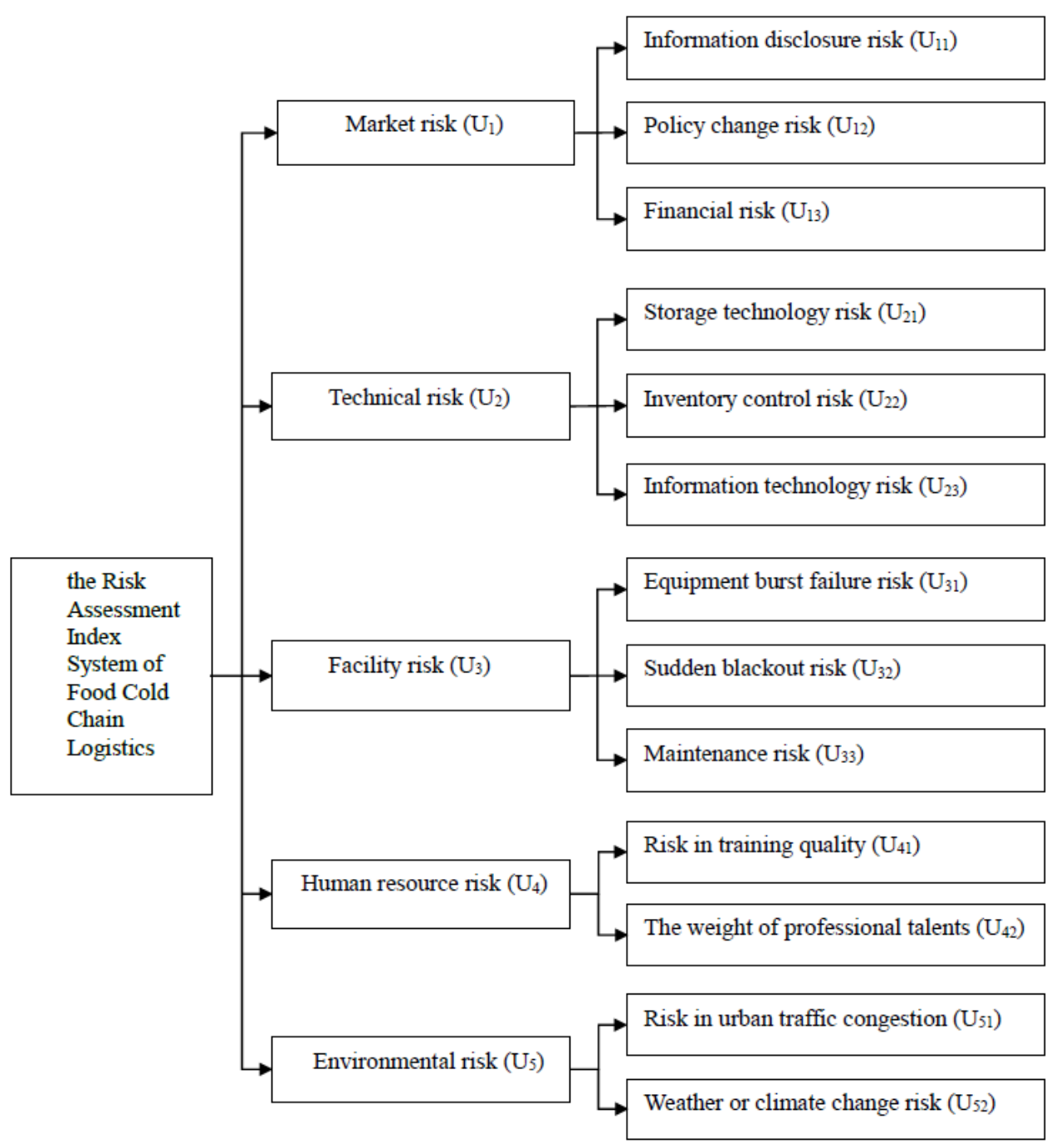

Fig. 1 the Risk Assessment Index System of Food Cold Chain Logistics 